\title{
An Accurate kTC Noise Analysis of CDS Circuits
}

\author{
Antonino Caizzone, Assim Boukhayma and Christian Enz \\ École Polytechnique Fédérale de Lausanne (EPFL) - Integrated Circuit Laboratory (ICLAB), Neuchâtel, Switzerland \\ antonino.caizzone@epfl.ch
}

\begin{abstract}
This work presents an analytical analysis of the thermal noise in widely used correlated double-sampling (CDS) circuits. The objective is to provide designers with simplified noise formulas essential for the design and optimization of such blocks. The obtained analytical results are confirmed with SpectreRF noise, SpectreRF transient noise and ELDO transient noise simulations.
\end{abstract}

Keywords-Noise, CDS, kTC, PSD, SNR, offset, thermal.

\section{INTRODUCTION}

In IC design, reducing the low-frequency noise and DC offset has become mandatory given the reduction of the dynamic range resulting from the suppy voltage reduction. Several circuit techniques have been proposed as solutions to the abovementioned challenge, such as the autozero (AZ), the correlated double-sampling (CDS) and the chopper stabilization (CHS) [1]. CDS is a very effective technique for offset and low frequency noise cancellation. Similarly to AZ, CDS is a sampled-data technique, except that the signal is sampled twice and eventually the difference between these two samples is taken, within the same clock period. Mathematically, the CDS transfer function imposes a zero at the origin of frequency which cancels any offset and drammaticaly reduces the low frequency noise [1]. Historically, the CDS technique has been originally introduced to reduce the noise generated in charged-couple devices (CCDs) [2]. Progressively, CDS has been extensively used in sampled-data systems and switched-capacitor (SC) circuits, in an increasing variety of possible applications, such as CMOS image sensors (CIS) [3] or analog front-end (AFE) for biomedical applications [4].

Despite CDS circuits reduce dramatically the low frequency noise, they remain limited by circuit non-idealities. Indeed, CDS circuits are mainly limited by analog switches nonidealities (on and off resistances), charge injection and thermal noise, the latter usually referred to as kTC noise [5]. The sampled noise voltage variance (kT/C) is, by definition, inversely proportional to the capacitance. On the contrary, both power and silicon area are directly proportional to the capacitance, resulting into a noise/power-area trade-off [6]. From a design perspective, it is necessary to be fully aware of the capacitances ultimately limiting the circuit performance. kTC noise analysis of SC circuits is never a simple task, considering that the noise transfer function, for these circuits, changes in time. Modern CAD simulators are useful in the estimation of the overall noise features, despite they require a very high accuracy set-up, resulting into extremely long simulations. Moreover, they don't provide simple analytical expressions to optimize the SC circuit noise.

In this perspective, this paper presents a simple and comprehensive kTC noise analysis of three different CDS circuits. It is organized as follows: Section II overviews the three proposed CDS structures, namely, a fully-passive CDS, a voltage bufferbased CDS and an amplifier-based CDS. Section III presents a detailed kTC noise analysis of each proposed CDS circuit. Section IV presents the noise simulations, showing a good match with the analytical calculations of Section III, and compares the three proposed structures. Section V concludes the paper.

\section{CDS CIRCUITS}

The three proposed CDS circuits are depicted in Fig. 1, namely, a fully-passive CDS, Fig. 1(a), a voltage buffer-based CDS, Fig. 1(b), and an amplifier-based CDS, Fig. 1(c). The circuit of Fig. 1(a) embeds passive elements only. Referring to the timing diagram shown in Fig. 1(d), a generic input signal voltage, $V_{1}$, is first sampled on the capacitor $C_{1}$, at the end of phase $\phi_{1}$. Secondly, at the end of phase $\phi_{2}$, a second input signal voltage, $V_{2}$, is sampled on the capacitor $C_{2}$. During the third phase $\phi_{3}$, the charge conservation principle leads to an output voltage equal to

$$
V_{\text {out }}=\frac{V_{1} C_{1}-V_{2} C_{2}}{C_{1}+C_{2}}=\frac{V_{1}-V_{2}}{2},
$$

assuming that $C_{1}=C_{2}$. Eq. (1) results into a CDS action, with a loss equal to two. From now on, we will refer to the fully passive CDS of Fig. 1(a) as $C D S_{1}$.

The circuit depicted in Fig. 1(b) consists of a voltage bufferbased CDS. Referring to the timing diagram shown in Fig. 1 (d), two independent input signals $V_{1}$ and $V_{2}$ are sampled on $C_{1}$ and $C_{2}$, respectively, during phase $\phi_{1}$. In the next phase $\phi_{2}$ the charge previously stored in $C_{1}$ and $C_{2}$ is transferred, through the two voltage buffer of gain $A_{v}$, to the output capacitor $C_{3}$. At the end of this phase, the voltage across $C_{3}$ is

$$
V_{\text {out }}=A_{v}\left(V_{1}-V_{2}\right) \text {. }
$$

Referring to (2), $A_{v}$ is the gain of the voltage buffer. In case of source follower stages, featuring body effect, $A_{v}=1 / n$, where $n$ is the slope factor (larger than one), this accounting for the body effect in MOS transistors, [7]. $A_{v}$ takes values closer to one for source to bulk connected devices. As in the previous case, Eq. (2) represents a CDS, with a loss accounting for the source follower non-idealities, i.e. $n>1$. From now on, we will refer to the CDS of Fig. 1(b) as $C D S_{2}$. 


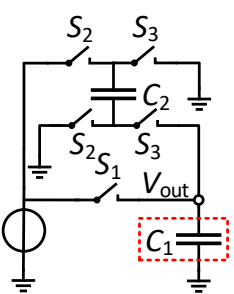

(a)

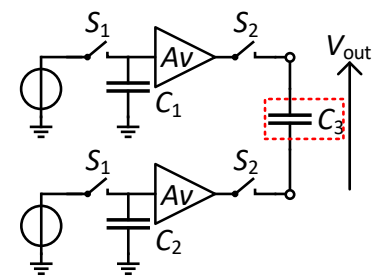

(b)

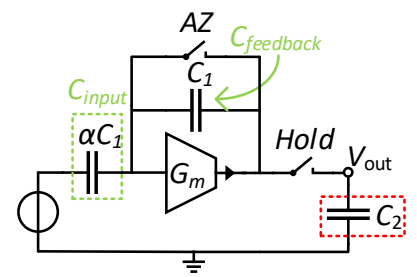

(c)

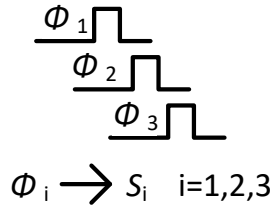

(d)

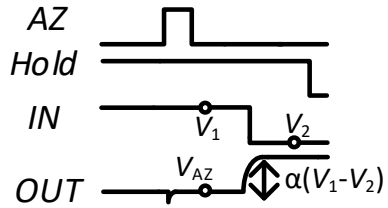

(e)

Fig. 1. Schematic of the three proposed CDS circuits with the timing diagrams: (a) fully-passive CDS, (b) voltage buffer-based CDS, (c) amplifier-based CDS, (d) timing diagram for (a) and (b), (e) timing diagram for (c).

Fig. 1(c) shows an amplifier-based CDS. Referring to the timing diagram of Fig. 1(e), the amplifier (transconductor) is first autozeroed. This phase, i.e. $A Z$, reduces the low frequency noise and the offset of the amplifier [1] and resets the feedback capacitor $C_{1}$. After this phase, in the scenario that the input signal, to the amplifier, toggles between the level $V_{1}$ and the level $V_{2}$, the amplifier output variation, i.e. $\Delta V_{\text {out }}$, results in

$$
\Delta V_{\text {out }}=\alpha\left(V_{1}-V_{2}\right),
$$

being $\alpha \cong-C_{\text {input }} / C_{\text {feedback }}$ the closed-loop gain. As in $C D S_{1}$ and $C D S_{2}$, Eq. (3) shows the difference of the input signal levels, so a CDS. From now on, we will refer to CDS of Fig. 1(c) as $C D S_{3}$.

\section{Noise CAlCUlation In CDS CiRCUits}

Objective of Section III is to derive the detailed kTC noise for each CDS circuit. It is important to remember that for a thermal noise source, having a constant Power-Spectral Density (PSD) $S_{n}$, its corresponding noise variance is equal to

$$
V_{n}^{2}=S_{n} \cdot N B
$$

where NB is the noise bandwidth, defined as

$$
N B=\left(1 / H_{n}^{2}(0)\right) \cdot \int_{0}^{\infty}\left|H_{n}(f)\right|^{2} d f .
$$

In (5), $H_{n}(f)$ is the noise transfer function. The noise transfer functions encountered in this work are of three types: $1^{s t}$ order low-pass (LP), $2^{\text {nd }}$-order LP (with zero) and $2^{\text {nd }}$-order band-pass (BP)

$$
\begin{gathered}
\left.H_{n}\right|_{1^{s t}}(f)=\left.\frac{1}{1+\frac{j f}{f_{c}}} \quad H_{n}\right|_{2^{n d} L P}(f)=\frac{1+\frac{j f}{f_{z}}}{1+\frac{j f}{f_{0} \cdot Q}+\left(\frac{j f}{f_{0}}\right)^{2}} \\
\left.H_{n}\right|_{2^{\text {nd } B P}}(f)=\frac{\frac{j f}{f_{0}}}{1+\frac{j f}{f_{0} \cdot Q}+\left(\frac{j f}{f_{0}}\right)^{2}}
\end{gathered}
$$

for which the NB is equal to

$$
\begin{aligned}
\left.N B\right|_{1^{s t}}=\left.\frac{\pi}{2} f_{c} \quad N B\right|_{2^{n d} L P} & =\frac{\pi}{2} f_{0} Q\left[1+\left(\frac{f_{0}}{f_{z}}\right)^{2}\right] \\
\left.N B\right|_{2^{\text {nd } B P}} & =\frac{\pi}{2} \frac{f_{0}}{Q},
\end{aligned}
$$

respectively. Eq. (7) will be extensively used throughout the work.
1) CDS1: due to the thermal noise originating from the onresistance of the switches, two uncorrelated kTC noise charge are injected in the switched capacitors $C_{1}$ and $C_{2}$ at the end of phase $\phi_{1}$ and $\phi_{2}$, respectively. In both phases $\phi_{1}$ and $\phi_{2}$, the on-resistor, $R_{o n}$, of the switches and the capacitor form a RC low-pass filter. Applying (4), (6) and (7) to this case and considering $S_{n}=4 k T R_{o n}$ results into noise variances equal to

$$
\left.V_{n C_{1}}^{2}\right|_{\phi_{1}}=\left.\frac{k T}{C_{1}} \quad V_{n C_{2}}^{2}\right|_{\phi_{2}}=\frac{k T}{C_{2}} .
$$

During the third phase $\phi_{3}$ the two capacitors share both their signal and uncorrelated noise charge, resulting into a noise voltage variance at the output node equal to

$$
\left.V_{n C_{1}}^{2}\right|_{\phi_{3}}=\frac{\left(C_{1}^{2} k T / C_{1}+C_{2}^{2} k T / C_{2}\right)}{\left(C_{1}+C_{2}\right)^{2}} .
$$

At the end of phase $\phi_{3}$, due to the switch $S_{3}$, an additional kTC noise charge, uncorrelated with the one generated in the previous phases, is injected in $C_{1}$ [5], resulting into an overall output voltage variance equal to

$$
V_{n_{\text {out }}}^{2}=\underbrace{\frac{\left(C_{1}^{2} k T / C_{1}+C_{2}^{2} k T / C_{2}\right)}{\left(C_{1}+C_{2}\right)^{2}}}_{\text {shared noise from } \phi_{1}, \phi_{2}}+\underbrace{\frac{k T}{C_{1}} \frac{C_{2}}{C_{1}+C_{2}}}_{\text {added noise from } \phi_{3}}=\frac{k T}{C_{1}} .
$$

2) $C D S 2$ : under the assumption that $C_{1}=C_{2}$ and exploiting the fully-differential structure, the small signal schematic of $C D S 2$, from a noise perspective, simplifies to the half circuit shown in Fig. 2(a). The total output noise voltage variance of the circuit of Fig. 1(b) is then simply equal to twice the output voltage variance of Fig. 2(a). With refer to the small signal schematic, three noise sources have to be accounted for: the on and off resistances of the two switches, $S_{1}$ and $S_{2}$, and the saturated MOS transistor in the source-follower. The noise coming from the off-resistor $R_{\text {off }}$ can be neglected as long as the corresponding time constant is much larger than the fraction of the period over which it is integrated (typically 1/3). The thermal noise (current) PSD of the saturated MOS transistor is $4 k T \gamma G_{m}$, where $\gamma \triangleq G_{m} R_{n}$ is the thermal noise excess factor ( $R_{n}$ is the input referred thermal noise resistance) [7] and $G_{m}$ the transconductance. Referring to Fig. 2(a), the voltagecontrolled current source $n G_{m} V_{2}$ accounts for the additional transconductance due to the body effect [7]. During phase $\phi_{1}$, $R_{1}$ is equal to the on-resistor, $R_{o n}$, while $R_{2}$ is equal to the offresistor, $R_{o f f}$. Evaluating the noise transfer functions related 


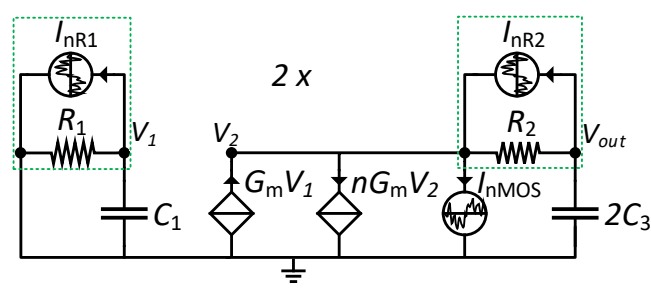

(a)

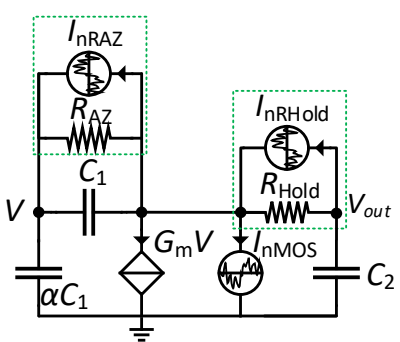

(b)

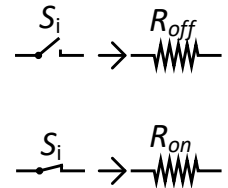

(c)

Fig. 2. Noise small signal schematic of CDS2 and CDS3: (a) CDS2, (b) CDS3, (c) switch equivalent model.

to the noise sources $I_{n M O S}, I_{n R 1}$ and $I_{n R 2}$ and applying (4), (6) and (7) leads to (considering the differential structure)

$$
\left.V_{n C_{1}}^{2}\right|_{\phi_{1}} \cong \underbrace{\frac{2 k T}{C_{1}}}_{S_{1} R_{o n}} \text {. }
$$

Repeating the same procedure during phase $\phi_{2}$ and exploiting again the differential structure results into a voltage variance on $C_{3}$

$$
\left.V_{n C_{3}}^{2}\right|_{\phi_{2}} \cong \underbrace{\frac{\gamma k T}{n C_{3}}\left(\frac{1}{1+n G_{m} R_{o n}}\right)}_{\text {MOS channel }}+\underbrace{\frac{k T}{C_{3}}\left(\frac{n G_{m} R_{o n}}{1+n G_{m} R_{o n}}\right)}_{S_{2} R_{o n}} .
$$

In this case, $R_{1}$ is equal to the off-resistor, $R_{o f f}$, while $R_{2}$ is equal to the on-resistor, $R_{o n}$. $C_{3}$ accounts for any additional parasitic contribute too, despite these have been neglected. Eventually, assuming that the noise on $C_{1}$ in $\phi_{1}$ and on $C_{2}$ in $\phi_{2}$ are uncorrelated, and that $G_{m} R_{\text {on }} \cong 0$, the overall kTC variance on the output capacitor $C_{3}$ is

$$
V_{n_{\text {out }}}^{2}=\left.A_{v}^{2} V_{n C_{1}}^{2}\right|_{\phi_{1}}+\left.V_{n C_{3}}^{2}\right|_{\phi_{2}} \cong \frac{2 k T}{C_{1}}+\frac{\gamma k T}{n C_{3}} \text {. }
$$

3) CDS3: this stage operates in two phases, namely, the $A Z$ phase and the amplification phase, during which the amplifier provides a closed-loop gain $\alpha$. In both phases, the overall kTC noise at the output of $C D S_{3}$ consists of two terms, both caused by the $\mathrm{AZ}$ and Hold switch resistance and the saturated MOS devices of the transconductor (OTA). First, the overall noise generated during the $A Z$ phase which is frozen in $\alpha C_{1}$ and eventually transferred to the feedback capacitor during the Amp phase. Secondly, the one generated in the amplification phase. Both noise variances add to the output, contributing to the overall kTC output thermal noise variance. Note that the frozen noise generated during the $A Z$ phase could be canceled by a second CDS stage. The noise sampled on $\alpha C_{1}$ at the end of the $A Z$ phase can be calculated from the schematic shown in Fig. 2(b). In this case, $R_{A Z}$ and $R_{\text {Hold }}$ represent the on-resistor, $R_{o n}$, of the switch $A Z$ and Hold, respectively. Evaluating the noise transfer functions related to the noise sources $I_{n M O S}, I_{n R A Z}$ and $I_{n R H o l d}$ and applying (4), (6) and (7) leads to

$$
\left.V_{n \alpha C_{1}}^{2}\right|_{A Z} \cong \underbrace{\frac{\gamma k T}{\alpha C_{1}+C_{2}}}_{\text {MOS channel }}+\underbrace{\frac{k T}{\alpha C_{1}} \frac{C_{2}}{\alpha C_{1}+C_{2}}}_{\text {Hold } R_{o n}} .
$$

Referring to (14), it can be shown that the noise contribution of $R_{A Z}$ is negligible with respect to the $R_{H o l d}$ one. Repeating the same procedure during the $A m p$ phase results to

$$
\left.V_{n C_{2}}^{2}\right|_{A m p} \cong \underbrace{\frac{\gamma k T\left(1+\alpha^{2}\right)}{\alpha C_{1}+C_{2}(1+\alpha)}}_{\text {MOS channel }}+\underbrace{\frac{k T}{C_{2}} \frac{\alpha C_{1}}{\alpha C_{1}+C_{2}(1+\alpha)}}_{\text {Hold } R_{\text {on }}} .
$$

In this case, $R_{A Z}$ represents the off-resistor, $R_{o f f}$, of the switch $A Z$, whilst $R_{H o l d}$ the on-resistor, $R_{o n}$. As in CDS2, the off-resistor contribution can be neglected. Assuming that the noise given by (14) and (15) are uncorrelated, the overall kTC variance at the output capacitor $C_{2}$ is then given by

$$
\left.V_{n_{\text {out }}}^{2} \cong \alpha^{2} V_{n \alpha C_{1}}^{2}\right|_{A Z}+\left.V_{n C_{2}}^{2}\right|_{A m p}
$$

As above-mentioned, at the end of the $A Z$ phase, the noise variance (14) gets frozen in the input capacitor, $\alpha C_{1}$, and simply added, multiplied by the square of the closed-loop gain, to the one generated in the next phase. In the above analysis, the parasitic capacitors are neglected. Note that the expression of the total output noise variance given by (16) includes the effect of the switches on-resistance which is usually neglected in the literature.

\section{Simulation Results}

In order to confirm the analytical noise calculations presented in Section III we have performed noise simulations. All the presented CDS circuits have been simulated by using SpectreRF Noise and Transient Noise simulations and Eldo Transient Noise simulations. SpectreRF Noise performs AC small-signal analysis, deriving the noise transfer functions, for all the noisy elements, and eventually integrating the result (multiplied by the PSD, as in (4)) over the chosen frequency span. This is the same approach as reported in the analytical analysis of Section III. Indeed, all the analytical formulas reported in Section III have been validated, for each phase, by the means of SpectreRF Noise simulations. On the contrary, both Eldo and SpectreRF Transient Noise simulations model each noise source as a sum of sinusoids over the frequency range of interest, with random phase, and with amplitude equal to the given noise PSD [8]. Transient noise simulations become particularly useful when the noise is large or the circuit highly nonlinear. Moreover, these simulations are the best ones to assess how the noise evolves in time, which means in the most realistic 


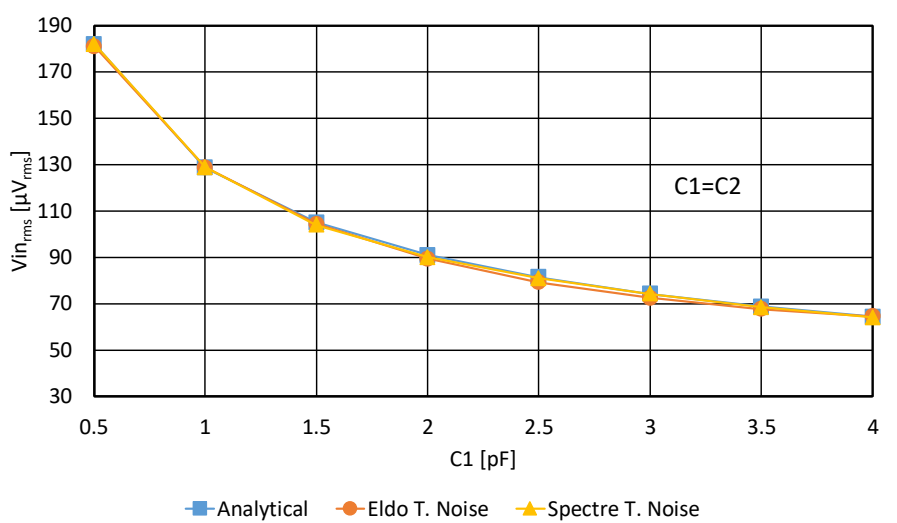

Fig. 3. Simulated and calculated RMS noise vs $C_{1}$ for CDS1.

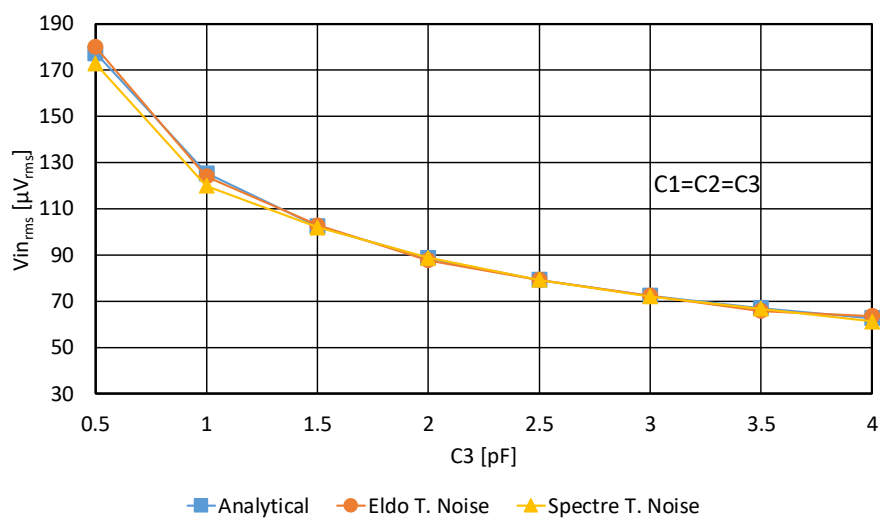

Fig. 4. Simulated and calculated RMS noise vs $C_{3}$ for CDS2.

condition. All the capacitors and switches of the circuits meet the condition $f_{t h, \max } \gg 1 /\left(2 \pi R_{o n} C\right)$, where $f_{t h, \max }$ is a simulation parameter setting the maximum noise frequency. The simulation results, for the three CDS circuits, are shown in Figs. 3 to 5: both SpectreRF and Eldo match very well with the calculated noise. Particularly, for $C D S 3$, Fig. 5 shows the effect of neglecting the switches on-resistance in the final computation, showing that they usually cannot be neglected. The noise is reported input-referred (output noise rms divided by the gain). The simulations are performed with $f_{t h, \max }=8 \mathrm{GHz}$, 100 noise simulations, $T=300 K, \gamma=1.5, n=1.2$ and $G_{m}=20 \mu S$.

\section{CONCLUSIONS}

A noise analysis of three different CDS circuits is presented. Both Eldo Transient noise and SpectreRF Transient and noise simulations show a very good match with the results obtained analytically. The fully passive features of CDS1 make it particularly suitable for ultra-low power applications, despite the signal-to-noise ratio (SNR) is affected by the intrinsic signal loss due to the charge sharing mechanism. Moreover, it is not affected by any signal saturation, the latter usually due to active elements. One of the main advantages of this implementation is that the SNR can be improved without limiting the input
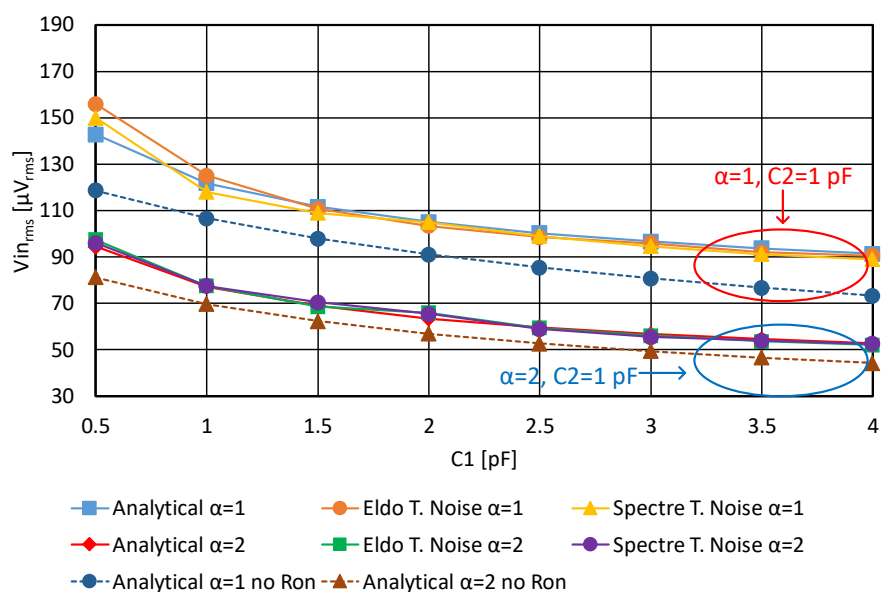

Fig. 5. Simulated and calculated RMS noise vs $C_{1}$ for CDS3.

signal range and only at the cost of more silicon area (larger capacitors). On the contrary, the amplifier-based CDS3 features an input-referred noise inversely proportional to the gain which depends directly on the size of the input capacitor. Hence, a lower noise is obtained at the cost of a lower input range, larger silicon area and power consumption. The advantage of CDS1 over CDS3 becomes even more obvious for values of $\gamma$ larger than two. CDS3 remains a good solution for combining amplification with CDS. The voltage buffer-based CDS2 shows roughly the same noise performance than CDS1 at the cost of more power, area and non-linearity.

In addition, the presented work highlights the impact of switches on-resistance which are usually neglected. This is even more true whenever the designer needs to minimize as much as possible the noise due to active elements.

\section{REFERENCES}

[1] C. Enz and G. C. Temes, "Circuit Techniques for Reducing the Effects of Op-amp Imperfections: Autozeroing, Correlated Double Sampling, and Chopper Stabilization." Proceedings of the IEEE, vol. 84, no. 11, pp. 1584-1614, Nov 1996.

[2] R. J. Kansy, "Response of a Correlated Double Sampling Circuit to 1/f Noise [generated in CCD arrays]." IEEE Journal of Solid-State Circuits, vol. 15 , no. 3, pp. 373-375, Jun 1980.

[3] A. Boukhayma, A. Peizerat, and C. Enz, "Noise Reduction Techniques and Scaling Effects Towards Photon Couting CMOS Image Sensors." Sensors, vol. 16, no. 4, p. 514, April 2016.

[4] A. Caizzone, C. Enz, and A. Boukhayma, "Comprehensive Noise Analysis in PPG Read-out Chains," 2017 International Conference on Noise and Fluctuations (ICNF), pp. 1-4, June 2017.

[5] A. Boukhayma and C. Enz, "A new method for kTC noise analysis in periodic passive switched-capacitor networks." 2015 IEEE 13th International New Circuits and Systems Conference (NEWCAS), pp. 1-4, June 2015.

[6] R. Castello and P. Gray, "Performance limitations in switched- capacitor filters," IEEE Transactions on Circuits and Systems, vol. 32, no. 9, pp. 865-876, September 1985.

[7] C. Enz and E. A. Vittoz, Charge-Based MOS Transistor Modeling: The EKV model for low-power and RF IC design. John Wiley \& Sons, Ltd, 2006.

[8] P. Bolcato and R. Poujois, "A new approach for noise simulation in transient analysis," [Proceedings] 1992 IEEE International Symposium on Circuits and Systems, vol. 2, pp. 887-890 vol.2, May 1992. 